\title{
Atención a la depresión generada durante la pandemia COVID 19
}

\section{Attention to depression generated during the pandemic COVID 19}

\author{
DOI: $10.46932 / s f j d v 2 n 5-045$
}

Received in: Oct 1st, 2021

Accepted in: Dec 30th, 2021

\section{Eduardo De La Fuente Rocha}

Último grado académico: Estancia Posdoctoral en el Instituto de Psicología Clínica y Social. México. 1994.

Maestría en Psicoterapia Junguiana. Instituto C. G. Jung de México Tercer Maestría. 1995

Maestría en Psicoterapia Gestalt. Instituto Mexicano de Psicoterapia Gestalt. Segunda Maestría 1993.

Doctor en Psicología Social. UNAM. 1990.

Maestría en Planeación UNAM. Primera Maestría. 1985

Institución actual: Profesor-Investigador de la Universidad Autónoma Metropolitana -Xochimilco.

Departamento de Educación y Comunicación. División de Ciencias Sociales y Humanidades.

Dirección: av. Gabriel macera 511, colonia del valle, delegación benito juarez, ciudad de méxico. C.p. 03100.

Correo electrónico: fuentee@correo.xoc.uam.mx edelafuente83@yahoo.com.mx

\section{RESUMEN}

El año 2020, se caracterizó por el impacto que, tuvo en la población, la pandemia del COVID-19. Dentro de los problemas de salud, destacan los generados por el aislamiento de la población qué cambió el comportamiento y la manera de desenvolverse tanto dentro del hogar como fuera de él. Al cambiar el estilo de vida, también cambió la calidad de ésta, afectando las relaciones humanas, generando desequilibrios emocionales que incidieron en un debilitamiento de la salud mental, propiciando miedo, estrés, ansiedad y depresión en la población, así como una disminución en la capacidad de un sano afrontamiento.

Este trabajo revisa, los problemas relacionados con la depresión y se hace una revisión cuidadosa de los niveles de intensidad en los que puede presentarse como consecuencia de los desequilibrios emocionales, cognitivos y valorativos que gestó la pandemia, proponiendo algunas acciones que permitan afrontar los procesos depresivos.

Palabras clave:Salud mental, Depresión, Desequilibrio, Aislamiento, Afrontamiento.

\begin{abstract}
ABSTACT
The year 2020 was characterized by the impact that the COVID-19 pandemic had on the population. Among the health problems, those generated by the isolation of the population stand out, which changed the behavior and the way of functioning both within the home and outside it. By changing the lifestyle, the quality of life also changed, affecting human relationships, generating emotional imbalances that led to a weakening of mental health, causing fear, stress, anxiety and depression in the population, as well as a decrease in the capacity for healthy coping.

This work reviews the problems related to depression and makes a careful review of the levels of intensity in which it can occur as a consequence of the emotional, cognitive and evaluative imbalances that the pandemic created, proposing some actions that make it possible to face the depressive processes.
\end{abstract}

Keywords: Mental Health, Depression, Imbalance, Isolation, Coping. 
El año 2020, se caracterizó por el impacto que, en diferentes aspectos, tuvo en la población, el problema ocasionado por una cepa viral llamada SARS- CoV-2 que invadió el planeta gestando la pandemia del COVID-19. Uno de los aspectos importantes que se presentó fue el detenimiento de las actividades de todo tipo, sociales, educativas, comerciales y administrativas, llevando a la población a vivir una vida de distanciamiento social y encierro. Una de las incidencias importantes, se dio en los trabajadores de la salud que atendían directamente a los pacientes enfermos del COVID-19, pues muchos de ellos fallecieron. Al cambiar el estilo de vida, también cambió la calidad de ésta, afectando las relaciones humanas y generando desequilibrios emocionales que incidieron en el debilitamiento de la salud mental, generando, en muchos casos, miedo, estrés, ansiedad y depresión en la población, así como una disminución en la capacidad de un sano afrontamiento.

A su vez, el aislamiento de los pobladores en sus casas trajo diferentes consecuencias psicológicas aunadas a otros daños, como la pauperización y el desempleo. La población, al cursar la pandemia ha experimentado diversos desequilibrios y efectos dañinos en las emociones colectivas (Valero, 2020), tales como: sentimientos de incertidumbre y de abandono, sentimientos de acoso, que han desencadenado ideaciones persecutorias, temores a nuevas enfermedades o a variaciones incontrolables de la enfermedad, sentimientos claustrofóbicos, conductas evasivas como puede ser el refugiarse en los aparatos tecnológicos, o en la bebida, etc.

También es importante señalar que el miedo social, al expandirse, abona a la discriminación de determinados sectores de la población, como por ejemplo la discriminación reportada en Estados Unidos y en Gran Bretaña en contra de las personas con ascendencia o aspecto asiático. (Russell, 2020), o la agresividad que este tipo de problemas suele generar en contra de poblaciones vulnerables, estigmatizándolas o desarrollando xenofobia en su contra. (Madhav, 2017).

Aunado a estos problemas, la economía de todos los países se ha visto afectada trayendo consigo el debilitamiento o la extinción de diversas empresas y negocios, provocando el despido de los trabajadores y la consecuente contracción de la economía familiar e individual.

En cuanto a las afectaciones psicológicas en la población, resulta fundamental tomarlas en cuenta, pues son fuente del desarrollo de múltiples enfermedades físicas y mentales, tales como la angustia colectiva o el desencadenamiento de alteraciones antes latentes en las personas, por ejemplo, el caso de los trastornos de personalidad antisocial, que se manifiestan durante el brote de la pandemia. Estos problemas, se presentan durante la pandemia, en su diseminación y aún, después de ella, pues se ocasionan fuertes desequilibrios mentales en la población por estrés postraumático. La alteraciones emocionales, son reacciones naturales que se presentan ante situaciones de peligro y cumplen con la función de promover en los individuos su adaptación, pero cuando rebasan ciertos niveles ya sea en intensidad, duración o 
frecuencia, generan en el grupo sensaciones de indefensión, obstaculizando el desempeño de las acciones de afrontamiento. ${ }^{1}$

En cuanto a las áreas que deben participar en la atención de la problemática de los desequilibrios mentales durante cada fase de la pandemia, es importante que se cubran tanto desde el ámbito médico como el psicológico, (Usher, 2020). Desde el punto de vista médico se ha querido atender este tipo de problemas con medicamentos. Sin embargo, es importante considerar que las alteraciones en el afecto pueden ser atendidas por los especialistas de la salud que se dedican a la psicología, a la psicoterapia y a todas aquellas ramas afines que tienen como finalidad restablecer el equilibrio psicológico de pacientes mediante diversas técnicas y tratamientos.

Uno de los problemas generales en la población ha sido la depresión, la que inicialmente se puede diluir mediante actividades físicas realizadas en casa, o promoviendo el contacto social, el cual, por el aislamiento obligado, se ha desarrollado de manera virtual por medio de videoconferencias, (Mendoza, 2020) mensajes de texto, correos electrónicos, redes sociales, llamadas telefónicas etc.

El apoyo mutuo para buscar solución a los problemas cotidianos y el construir ambientes emocionales agradables también contribuye al abatimiento de los desequilibrios emocionales. En caso de la falta de apoyo, se han generado desavenencias familiares cuando no la ruptura de las relaciones. La participación en actividades religiosas, a través de los medios electrónicos también ha coadyuvado a la prevención y a la disminución del estrés y de la depresión en situaciones de crisis (Villalba, 2012).

Los diversos tipos de pérdidas que ha tenido que enfrentar la población durante la pandemia, van desde la falta de una movilidad amplia, hasta la muerte de seres queridos en condiciones muy diferentes a las cotidianas, pues muchas de estas pérdidas se han tenido que enfrentar sin los procedimientos rituales que normalmente se practican en un duelo, como es el velorio o el entierro, junto con el acompañamiento y el apoyo moral y emocional de familiares y amigos, En múltiples casos, el último momento en el que han visto al familiar contagiado, ha sido durante su ingreso al hospital y el siguiente contacto, se ha dado al recibir las cenizas del mismo, lo que deja a los deudos con daños psíquicos y fijaciones por el proceso no resuelto.

Todos los tipos de pérdidas, desde las sociales y materiales hasta las afectivas más significativas, han gestado manifestaciones depresivas en la población (Torales, 2020). Tal depresión se ha presentado con diferentes intensidades o se puede observar en diversas manifestaciones, tales como problemas somáticos, trastornos del sueño, inestabilidad emocional, actitudes agresivas o de excesiva indiferencia, alteraciones en la percepción, en conductas inapropiadas o disfuncionales etc.

\footnotetext{
${ }^{1}$ Área Humana. Centro Clínico de Atención Psicológica. Afrontamiento psicológico del coronavirus. Guía de orientación emocional. Disponible en: https://www.areahumana.es/afrontamiento-psicologicocoronavirus/\#Lecturas-tiles-para-elafrontamiento-psicolgicoante-el-Coronavirus
} 
Se ha podido observar que un factor que influye en el incremento de la depresión es la exposición continua, que la población enfrenta, a la información relativa a la pandemia, muchas veces equívoca y en otras amenazante, debido a que muestra los avances de la pandemia, sus peligros los daños causados tanto nacionales como internacionales, lo cual hace que la población se sienta sobrepasada en su capacidad de afrontar. (Avittey,2020)

De acuerdo con el Dr. Francisco Ferre Navarrete, la depresión es:

"Es una enfermedad multifactorial - es decir en la que intervienen muchas circunstancias - que involucra el temperamento y los rasgos de personalidad como predisponentes, la exposición a situaciones vitales estresantes y la susceptibilidad biológica genéticamente determinada". (Ferre, 2007:5)

Existen diferentes expresiones emocionales, actitudes, conductas y pensamientos, por las que es posible darse cuenta de que una persona está entrando o está en depresión, y estas, se manifiestan a través de las propias declaraciones que cada vez hace con más frecuencia el sujeto. Algunos ejemplos de ellas son:

- $\quad$ Cambios emocionales bruscos y repentinos.

- Declaraciones repetidas en las que afirma estar experimentando muchos momentos de tristeza y continuos.

- Apatía e indolencia ante las propuestas de acción a las que le invitan otras personas y manifestaciones repetidas de no querer llevar a cabo ninguna actividad y de no esperar nada en la vida.

- $\quad$ Llanto frecuente sin causa aparente o llanto muy frecuente cuando habla de su desesperanza o de sus pérdidas.

- $\quad$ Sentimientos auto punitivos por no haber actuado adecuadamente en el momento oportuno declarando por ello, sentirse culpable y poco valioso.

- Cursar sentimientos fuertes de soledad y el mismo tiempo presentar una tendencia a aislamiento, al encierro y al ensimismamiento.

- $\quad$ Pérdida de la autoestima y de la seguridad en sí mismo.

- $\quad$ Sentimientos de intranquilidad constantes y/o irritabilidad.

- Hipersensibilidad disfuncional.

- $\quad$ Sentimientos de insuficiencia y temor de no ser capaz de realizar las actividades que antes llevaba a cabo con facilidad.

- $\quad$ Pérdida de la capacidad de disfrute.

- Rechazo a convivir y resistirse a participar en el intercambio social que hacen sus seres cercanos a través de la comunicación.

- $\quad$ Sensación continua de agotamiento y cansancio.

- Indolencia y/o abandono de las actividades laborales.

- $\quad$ Alteraciones en el dormir que puede ser: el tardarse en conciliar el sueño o despertar varias veces y después no poder fácilmente volver a dormir, o el sentir que el levantarse de la cama es un reto muy difícil de lograr.

- $\quad$ Pérdida de interés por la propia higiene, del cuidado corporal y del arreglo personal.

- Desequilibrios alimentarios, como es, el comer demasiado o el dejar de alimentarse.

- Manifestaciones verbales por las que expresa a los que le rodean que, para él, sería mejor morir. 
- $\quad$ Aumento de las preocupaciones relativas a lo que está sucediendo, sobre lo que le puede suceder a sus seres cercanos y/o en contraste, indiferencia de lo que le pueda pasar a él, expresando que le da igual si muere o si continúa con vida.

- Bloqueos en la atención. Aumento de la distraibilidad. Notoria pérdida de memoria sobre datos que normalmente retiene con facilidad. Desorientación.

- Incremento en la tendencia a tener pensamientos oscuros sobre sí mismo o sobre lo que le espera en el futuro.

Aunque todas las personas podemos cursar en algún momento estados depresivos existen en las personas, situaciones y factores que hacen que unos sujetos sean más propensos a entrar en depresión que otros. Ejemplo de personas que pueden tener un mayor riesgo de entrar en depresión son:

- Los sujetos que constantemente están expuestos a situaciones estresantes.

- $\quad$ Personas que desde tiempo atrás han experimentado estrés.

- Sujetos que han sufrido fuertes pérdidas en la vida o en el momento de la pandemia las están cursando. Las pérdidas pueden ser de libertad, de salud económicas, etcétera.

- Sujetos que en su historia familiar tienen parientes cercanos que han sufrido depresión.

- Personas que portan desde la infancia experiencias altamente dolorosas que tuvieron que superar con mucha dificultad.

- $\quad$ Sujetos con duelos anteriores no resueltos

- Mujeres encinta o mujeres que experimentan fuertes cambios hormonales.

- $\quad$ Personas que tienen problemas importantes o graves de salud ya sean crónicos o recientes o por enfermedades terminales.

- Sujetos con una estructura psíquica débil cuyo equilibrio puede romperse ante una situación estresante.

- $\quad$ Personas que su historia han cursado por depresiones en varias ocasiones saliendo de ellas con dificultad.

- $\quad$ Sujetos que han sufrido un fuerte desarraigo de sus lugares de origen.

- $\quad$ Personas que han sido apartadas de sus gentes queridas.

Pueden presentarse factores desencadenantes de la depresión en sujetos propensos a la depresión, tales como:

- $\quad$ Propensión fisiológica o hereditaria

- $\quad$ Problemas sociales y muy especialmente de salud y de riesgo a perder la vida

- Daños económicos

Los estados depresivos, como ya se anticipó, pueden presentarse de forma más leve o profunda según sea la fuerza del impacto que cause en cada sujeto y pueden estar potenciados por factores personales sociales y culturales. Para un sujeto en particular, también se pueden presentar factores que intensifiquen la depresión, como son las enfermedades físicas y psíquicas preexistentes en la persona, la pérdida del empleo o la irritabilidad que provocan las desavenencias en el grupo con el que se convive. 
La depresión puede presentarse de forma más alarmante y con posibles consecuencias dañinas. De acuerdo con el manual de psicodiagnóstico MSD, los procesos depresivos que pueden ser considerados como patológicos graves son: el trastorno depresivo mayor, el trastorno depresivo persistente y otros trastornos depresivos que pueden ser especificados o no especificados. (DSM V, 2013).

Cuando aparecen situaciones que pueden favorecer el desarrollo de estados depresivos es importante atenderlos desde un inicio para que se abatan sus efectos y secuelas pues cuando éstos que vuelven excesivos para él sujeto pueden llegar a tener consecuencias graves tanto para él como para la sociedad.

Es por ello por lo que es necesario, además de proponer estrategias globales, impulsar de manera positiva propuestas específicas de afrontamiento que pueden ser precisamente orientaciones para poder decidir cuándo cada uno puede participar y en qué nivel para el abatimiento del problema de depresión, especialmente en los casos iniciales y cuando debe ser remitido a los especialistas para que tengan una atención oportuna. De esta manera se pretende brindar una orientación tanto individual como familiar y comunitaria para la atención del problema de la depresión disminuyendo sus efectos y secuelas.

Es importante saber, que la atención y el apoyo que se le puede brindar a una persona deprimida tendrán un efecto positivo en mayor o menor grado, dependiendo de los antecedentes antes mencionados. Cuando el sujeto ha cursado en diversas ocasiones por estados depresivos intensos, la probabilidad de lograr una recuperación completa disminuye. Sin embargo, el apoyo oportuno del personal adecuado, siempre aporta beneficios al deprimido disminuyendo sus malestares y su sufrimiento, así como las alteraciones conductuales, emocionales, cognitivas y físicas generadas por el proceso de depresión.

Cuando una persona cercana al deprimido o un profesional, se avoca a brindar apoyo al sujeto que cursa este problema, debe saber que se enfrentará a momentos difíciles en los que no estará entendiendo el proceso o le faltará claridad para saber si está brindando verdaderamente un apoyo adecuado o suficiente. Este trabajo busca el dar orientación a los que intervienen en el apoyo para tener cierta claridad en cómo, dónde y quienes pueden apoyar en forma más eficiente al sujeto.

La persona interesada en brindar este tipo de apoyos deberá contar con un concepto claro de lo que significa la depresión y deberá ser capaz de reconocer sus manifestaciones. También deberá saber cuándo acudir a personas especializadas y aprender a seguir sus indicaciones durante el tratamiento. La ayuda al paciente debe mantener la suficiente cercanía para que el paciente se sienta apoyado, pero sin llegar a la invasión ni a la sobreprotección. De esta manera la ayuda resultará más eficaz. Los casos en el que el paciente presente repetidamente ideas mórbidas y de suicidio, deberán ser canalizados con los especialistas para evitar un daño irreparable. 
A continuación, se plantea una estrategia para aclarar los diferentes tipos y niveles de intervención que se pueden llevar a cabo con un sujeto, que presenta un cierto grado de depresión. Para ello, se distinguen los tipos de daño que puede causar la depresión, como son: los desequilibrios físicos, los emocionales, los cognitivos y los valorativos. En cada uno de ellos se distinguen tres niveles de intensidad y se sugieren procedimientos a seguir con el sujeto deprimido en cada caso.

De manera general cuando no se trata de un periodo de mucha tensión sino de un periodo normal, es natural que se den procesos depresivos menores, en este caso, las personas pueden estar tristes, preocupadas o ansiosas por los eventos cotidianos que viven. Cuando el nivel de depresión es medio, los eventos llegan a sobrepasar a la persona saliéndose de su control y creando estados patológicos parciales. En los casos de depresión grave el deprimido ha perdido la funcionalidad física y mental de manera tal que, si no son atendidos oportunamente, pueden convertirse en procesos crónicos o inclusive llevar al sujeto a formas parciales de destrucción o a la muerte.

Existen entonces diversos niveles o grados de la depresión. Específicamente, entre los desequilibrios físicos y corporales que cursa una persona en depresión cuando el desequilibrio es menor, se observa que dicho sujeto puede estar dejando de tener interés en la realización de sus actividades cotidianas, sentirse cansado, o puede sentirse sin energía. Cuando el nivel es medio, puede el sujeto comenzar a alentar sus movimientos, dejar de bañarse, perder el apetito o comer en exceso. Puede tener pérdida o trastornos en el sueño, ya sea que presente insomnio o esté durmiendo demasiado. También puede presentar problemas sexuales o sentir dolores de cabeza o molestias en otras partes del cuerpo. Cuando el nivel de depresión es mayor, el sujeto desarrollará conductas corporales de alto riesgo, dañinas o auto destructivas, e incluso intentará suicidarse.

En cuanto a los desequilibrios emocionales que presenta un deprimido, cuando el problema es menor, manifestará alteraciones en los estados emocionales, con eventos intermitentes de angustia, tristeza, miedo y/o enojo. Cuando el nivel de alteración es medio el deprimido cursará por estados intensos de inquietud. Podrá desarrollar una tendencia constante a reaccionar con irritación. Se le percibirá agitado con sentimientos de frustración. En este nivel se perderá el interés por la experimentación de situaciones placenteras. Cuando el nivel de depresión es mayor, se presentarán altos niveles de culpa, de desesperanza, de autorreproches, con sentimientos profundos de frustración, generando estados intensos de angustia, tristeza y/o enojo.

En cuanto al desarrollo cognitivo, las depresiones menores pueden propiciar distracción, baja atención, olvidos frecuentes. Las depresiones de nivel medio pueden ocasionar dispersión de los pensamientos y falta de concentración en lo que se está realizando. Se perderá la capacidad de ejercer con éxito las funciones mentales superiores como son, el razonamiento, las funciones de la memoria, la 
atención, el análisis y la síntesis. En los casos de depresión mayor habrá una fuerte fijación de los pensamientos a situaciones pasadas. Al agravarse podrá venir la pérdida parcial, total, temporal o permanente de la conciencia del espacio y del tiempo en el que el sujeto vive.

En cuanto al aspecto valorativo, las consecuencias de la depresión en la persona que la padece, cuando ésta es menor, la llevarán a sentir que su autoestima es baja; en un nivel medio de depresión, el sujeto, dejará de tomar decisiones, manifestará poca valoración de los elementos y personas que le rodean, e incrementará la pérdida del interés en las relaciones sociales y en el cuidado de sí mismo. En los casos de depresión mayor, el sujeto se sentirá inútil, mostrará excesivo desinterés por realizar actividades, cursará una grave pérdida de la autoestima y comenzará a intensificar sus ideaciones mórbidas que pueden llevarlo al suicidio. Estos casos deben ser atendidos con prioridad (Reger, (2020).

Los desequilibrios psíquicos desarrollados en la población por la pandemia, cuando no son graves, pueden ser paleados por los mismos familiares y allegados al paciente, manteniendo siempre el autocuidado de la estabilidad emocional en las personas que apoyan.

Como propuesta general para tener una participación cuidadosa en la atención de los pacientes depresivos se propone que en los niveles de menor depresión se trabaje con una terapia conversacional qué pueden llevar a cabo los expertos en salud mental como son los psicoterapeutas. Este servicio puede ser apoyado por ejercicios de relajación o con sustancias naturales relajantes, que apoyen el proceso psicoterapéutico. Para los pacientes de nivel medio, es necesario que el proceso psicoterapéutico se acompañe del apoyo médico, pues son ellos quienes podrán administrar las dosis adecuadas de medicamentos antidepresivos acordes con cada caso particular. En el caso de los pacientes deprimidos de nivel profundo, en general y sobre todo a partir del momento en que manifiestan ideaciones suicidas, deberán ser internados para ser atendidos en instituciones especializadas, en donde podrán recibir tanto el apoyo médico como el apoyo psicoterapéutico. La manera como se administrarán ambos, será acorde al caso particular de cada paciente con el que se esté trabajando.

Las estrategias de orientación para el afrontamiento antes planteadas pueden complementarse con las otras formas de intervención ya antes señaladas, que ayudan mucho para abatir el problema, como son:

Proponer estrategias de afrontamiento globales para la población y apoyarlas positivamente. Una de ellas puede ser la difusión de orientaciones sobre los diversos tópicos relacionados con los desequilibrios emocionales gestados en la pandemia, presentadas de manera accesible al público. Un ejemplo de ello es este artículo, en el que se dan orientaciones generales que pueden ubicar a la población en el lugar y en la acción adecuada para el abatimiento de los diversos problemas de depresión y que además le pueden dar un marco de referencia para saber cuándo intervenir y cuándo derivar los casos hacia los especialistas, propiciando con ello una atención oportuna y adecuada. La promoción de las 
formas de orientación puede hacerse tanto a nivel individual como familiar o comunitario. Es importante reconocer la labor de los sistemas digitales que trabajan en apoyo al restablecimiento de la salud mental, por lo que se recomienda que el contenido de esta propuesta pueda difundirse a través de las redes que coadyuvan al sostenimiento de la salud en general y del equilibrio psíquico de la población en particular.

Todos los problemas emocionales cuando los familiares o amistades de un sujeto los comienzan a detectar, deben ser validados y tomados en cuenta. Con ello se evitará el que crezcan y se vuelvan problemas más graves. Los familiares y amistades del sujeto que comienza a sufrir de depresión pueden intervenir en un inicio, acercando al sujeto que padece la depresión a los apoyos técnicos y profesionales que le habrán de ayudar en la recuperación de un estado emocional equilibrado. Simultáneamente, las personas que participen en este tipo de apoyos deberán estar atentos a sus propios estados emocionales puesto que una exposición prolongada por la atención a este tipo de desequilibrios puede llegar a contaminar a los profesionales o personas cercanas que intervienen en la ayuda del paciente.

Es importante recordar que es tan valioso el apoyo médico como el psicológico. Los medicamentos en casos difíciles resultan de gran ayuda para estabilizar al paciente y así, permitir un mejor trabajo terapéutico con él. Los especialistas en la salud mental pueden ser psicólogos psicoterapeutas, y todas aquellas personas cuyo trabajo está relacionado con el restablecimiento del equilibrio psicológico y con la aplicación de técnicas y procedimientos que coadyuven en este proceso de mejoría.

También resulta de gran ayuda mantener el contacto social, la población ha aprendido a hacerlo a través de los medios electrónicos como son las videoconferencias las redes sociales y las llamadas telefónicas. Lo mismo puede decirse del beneficio que acarrea el apoyo mutuo pues ayuda a la construcción de ambientes emocionalmente agradables.

Se ha elaborado el presente trabajo con el fin de apoyar a la población durante el proceso de la pandemia ocasionada por el COVID 19, brindándole una orientación general que le permita saber que hacer en los casos de sujetos que presenten depresión. 


\section{BIBLIOGRAFÍA}

Área Humana. Centro Clínico de Atención Psicológica. Afrontamiento psicológico del coronavirus. Guía de orientación emocional. Disponible en: https://www.areahumana.es/afrontamientopsicologicocoronavirus/\#Lecturas-tiles-para-el-afrontamiento-psicolgicoante-el-Coronavirus

Asociación Americana de Psiquiatría, (2013) "Guía de consulta de los criterios diagnósticos del DSM 5". Arlington, VA. Washington, D.C.

Ayittey FK, Ayittey MK, Chiwero NB, Kamasah JS, Dzuvor C. (2020) "Economic impacts of Wuhan 2019-nCoV on China and the world". J MedIcal Virology. 2020;92 https://doi.org/10.1002/jmv.25706

Federación mundial para la salud mental. (2010) "Enfermedad mental y suicidio" F.M.S.M. USA

Ferre F. (2007) “Afrontar la depresión”. Fundación Arpegio. Madrid

Junta de Andalucía. Málaga (2013) "Guía de autoayuda para la depresión y los trastornos de ansiedad". Servicio Andaluz de Salud.

Junta de Andalucía. (2013) "Guía de autoayuda - Cómo afrontar el duelo". Servicio Andaluz de Salud. Málaga

Madhav, N., Oppenheim, B., Gallivan, M., Mulembakani, P., Rubin, E., et. al. (2017). "Pandemics: Risks, Impacts, and Mitigation". In: Disease Control Priorities (third edition): Volume 9, Disease Control Priorities, edited by D. T. Jamison, H. Gelband, S. Horton, P. Jha, R. Laxminarayan, C. N. Mock, R. Nugent. Washington, DC: World Bank.

Mendoza Velásquez J. (2020). Impacto de la COVID-19 en la salud mental. Disponible en: https://espanol.medscape.com/verarticulo/5905131.

Reger MA, Stanley IH, Joiner TE. (2020) Suicide Mortality and Coronavirus Disease 2019-A Perfect Storm?. JAMA Psychiatry.. doi:10.1001/jamapsychiatry.2020.1060

Russell A. (2020) The Rise of Coronavirus Hate Crimes. The New Yorker. Available from: URL: https://www.newyorker.com/news/letter-from-the-uk/the-rise-ofcorona virus-hate-crimes

Torales J, O'Higgins M, Castaldelli-Maia JM, Ventriglio A. (2020) The outbreak of COVID-19 coronavirus and its impact on global mental health. Int J Soc Psychiatry. 20764020915212. doi: 10.1177/0020764020915212.

Usher K, Durkin J, Bhullar N. (2020) The COVID-19 pandemic and mental health impacts. International Journal of Mental Health Nursing, doi: 10.1111/inm.12726

Valero-Cedeño NJ, Mina-Ortiz JB, Veliz-Castro TI, Merchán-Villafuerte KM, Perozo-Mena AJ. COVID19: La nueva pandemia con muchas lecciones y nuevos retos. Revisión Narrativa. Kasmera. doi:10.5281/zenodo.3745322

Villalba ME, Cots I, Romero Aguilar N. (2012) ¿Las Creencias Religiosas condicionan el afrontamiento de la enfermedad y muerte de un familiar? Evidentia: Revista de Enfermería basada en evidencia,; vol. 9 (39): 10. 